\title{
ON THE CONTINUITY OF THE WIENER-HOPF FACTORIZATION OPERATION
}

\author{
MICHAEL GREEN ${ }^{1}$ AND BRIAN D. O. ANDERSON ${ }^{1}$
}

(Received 1 November 1985; revised 1 April 1986)

\begin{abstract}
The problem of passing from an $L_{\infty}$ function to a Wiener-Hopf factorization is considered. It is shown that a small $L_{\infty}$ perturbation which does not change the factorization indices will lead to small $L_{p}(1<p<\infty)$ perturbations in the Wiener-Hopf factors, but can lead to large $L_{\infty}$ perturbations, unless the derivatives are controlled during the perturbation.
\end{abstract}

\section{Introduction}

The technique of Wiener-Hopf factorization, which will be defined in Section 3, has a wide variety of applications. These include the classical applications to the prediction theory of stochastic processes $[12,14,15]$ and the solution of integral and convolution equations $[7,9]$. When applied to non-negative Hermitian matrix functions the technique is termed spectral factorization because of its long association with stochastic theory $[12,15]$, but also has other applications including the solution of linear quadratic control problems [3]. Another sub-class of Wiener-Hopf factorization is canonical factorization, which includes spectral factorization. This technique has been applied to the determination of system stability via passivity theory [5]. More recently, the general Wiener-Hopf factorization technique has been applied to the identification of errors-in-variables models [10].

A question which naturally arises is the extent to which the operation of Wiener-Hopf factorization is continuous. It is known that if a matrix function $A$

\footnotetext{
${ }^{1}$ Department of Systems Engineering, Research School of Physical Sciences, Australian National University, G.P.O. Box 4, Canberra, A.C.T., 2601, Australia.

(c) Copyright Australian Mathematical Society 1987, Serial-fee code 0334-2700/87
} 
has a canonical factorization and another function $B$ is close, in $L_{\infty}$ norm, to $A$, then the factors of $A$ and $B$ will be close in $L_{p}$ norm, $1<p<\infty[7,2,1]$. However in many applications, particularly in control system theory, it is the $L_{\infty}$ norm which is most important [6]. It is therefore desirable to know conditions which will ensure that the operation of Wiener-Hopf factorization is continuous in $L_{\infty}$ norm. This question has been tackled for the special case of spectral factorization in [1], where it is shown that the derivatives of the matrices to be factored must be bounded in $L_{2}$ norm. Moreover an example shows that the factors may be very far apart in $L_{\infty}$ norm when the derivative condition is not satisfied.

It is the aim of this paper to extend these results first to canonical factorization in Section 3 and then to general Wiener-Hopf factorization in Section 4. Further motivation for the results in terms of their application significance will also be given. Section 2 will contain definitions of the various norms and spaces which will be used.

\section{Spaces, norms and notation}

Denote by $\mathbf{C}^{n \times n}$ the space of complex $n \times n$ matrices and any induced norm on $\mathrm{C}^{n \times n}$ by $|\cdot|$. A matrix function on the unit circle is a Lebesgue measurable mapping $M$ from $[0,2 \pi]$ to $\mathbf{C}^{n \times n}$, taking $\omega \in[0,2 \pi]$ to $M\left(e^{J} \omega\right) \in \mathbf{C}^{n \times n}$. We define the spaces $L_{p}, 1 \leqslant p \leqslant \infty$ of matrix functions as follows: For $1 \leqslant p<\infty$, let

$$
\begin{aligned}
\|M\|_{p} & =\left(\frac{1}{2 \pi} \int_{0}^{2 \pi}\left|M\left(e^{j \omega}\right)\right|^{p} d \omega\right)^{1 / p} \\
\|M\|_{\infty} & =\operatorname{ess} \sup \left|M\left(e^{j \omega}\right)\right|
\end{aligned}
$$

$L_{p}$ is the space of matrix functions whose corresponding norm is finite, i.e. $M \in L_{p}$ if and only if $\|M\|_{p}<\infty$. The $L_{p}$ spaces are Banach spaces, and $L_{2}$ is a Hilbert space. Moreover $L_{j} \subset L_{i}$ for $i<j$ and for $M \in L_{p}, 1 \leqslant p<\infty$ we can write the Fourier series, convergent in $\|\cdot\| p$

$$
M\left(e^{j \omega}\right)=\sum_{k=-\infty}^{\infty} M_{k} e^{j \omega k}
$$

where $M_{k} \in \mathbf{C}^{n \times n}$

The space $C$ denotes the space of continuous matrix functions. The Wiener algebra $W$ of matrix functions consists of matrix functions of the form (2.2) for 
which

$$
\|M\|=\sum_{k=-\infty}^{\infty}\left|M_{k}\right|
$$

is finite. $W$ is an algebra under multiplication of matrix functions. It is easy to verify that $W \subset C \subset L_{\infty}$.

For any matrix function $M$ of the form (2.2), define the projection operator $P_{+}$ by

$$
P_{+}(M)\left(e^{j \omega}\right)=\sum_{k=0}^{\infty} M_{k} e^{j \omega k}
$$

and the projection $P_{-}$by

$$
P_{-}=I-P_{+}
$$

If $Q$ is a linear operator on a Banach space $\left(B,\|\cdot\|_{B}\right)$, then the norm of $Q$, denoted $\|Q\|$ is defined by

$$
\|Q\|=\sup _{X \in B} \frac{\|Q x\|_{B}}{\|x\|_{B}}
$$

We shall use the fact later that $P_{+}, P_{-}$are bounded linear operators on $W$ and on $L_{p}, 1<p<\infty[2,7]$.

Let $S$ be any of the spaces $L_{p}, W, C$ and define $S^{+}$(resp. $S^{-}$) to be the image space of $P_{+}$(resp. $P_{-}$) acting on $S$. Also define $G S, G S^{+}, G S^{-}$to be the group of elements in $S, S^{+}, S^{-} \oplus I$ which have inverses, i.e. $M \in G S\left(G S^{+}, G S^{-}\right)$if $M$ and $M^{-1}$ are in $S\left(S^{+}, S^{-} \oplus I\right)$. The classic theorem of [13] states that if $M \in S$, then $M \in G S$ if and only if det $M\left(e^{j \omega}\right) \neq 0$ for all $\omega$. Furthermore, if $M \in S^{+}$ (resp. $S^{-}$) we can regard it as the evaluation on $|z|=1$ of a matrix function of $z$ which is analytic in and continuous up to the boundary of $|z| \leqslant 1$ (resp. $|z| \geqslant 1 \cup \infty$ ), and then $M \in S^{+}$(resp. $S^{-}$) is in $G S^{+}$(resp. $G S^{-}$) if and only if $\operatorname{det} M(z) \neq 0,|z| \leqslant 1$ (resp. $|z| \geqslant 1 \cup \infty$ ).

\section{Continuity of canonical factorization}

In this section we first briefly review canonical factorization, the material being taken from [5], [7] and [9], and then present two continuity results.

A matrix function $M \in G W$ is said to admit a (left) canonical factorization in case

$$
M=M_{+} M_{-},
$$

where $M_{ \pm} \in G W^{ \pm}$and $M_{-}(\infty)=I$. 
Define the following linear operators:

$$
\begin{aligned}
T_{M}(X) & =P_{+}(X M)+P_{-}(X), \\
R_{M}(X) & =P_{+}(X)+P_{-}(M X), \\
\hat{T}_{M}(X) & =P_{+}\left(M^{-1} X\right)+P_{-}(X), \\
\hat{R}_{M}(X) & =P_{+}(X)+P_{-}\left(X M^{-1}\right) .
\end{aligned}
$$

THEOREM A. A matrix function $M \in G W$ admits a left canonical factorization if and only if the operators $T_{M}, R_{M}$ are invertible on $G W$. In this case $\hat{T}_{M}, \hat{R}_{M}$ are also invertible and the canonical factors $M_{ \pm}$are given by

$$
\begin{aligned}
M_{+} & =\hat{T}_{M}^{-1}(I), \\
M_{-} & =\hat{R}_{M}^{-1}(I), \\
M_{+}^{-1} & =T_{M}^{-1}(I), \\
M_{-}^{-1} & =R_{M}^{-1}(I),
\end{aligned}
$$

where $I$ is the identity matrix function.

Note that the operators used in $[1,5]$ are $X-P_{+}(X Z), X-P_{-}(Z X)$ with $M=I-Z$. These operators are entirely equivalent to $T_{M}, R_{M}$.

We can more generally, and more conveniently from a continuity point of view, consider what is called generalized canonical factorization [4], or factorization in $L_{p}$. We enlarge the space of matrix functions $M$ which can be factored from $G W$ to $G L_{\infty}$, and correspondingly enlarge the space in which the factors lie from $G W^{ \pm}$to $M_{+} \in L_{q}^{+}, M_{+}^{-1} \in L_{p}^{+}, M_{-} \in L_{p}^{-}, M_{--}^{-1} \in L_{q}^{-}$, where $p^{-1}+q^{-1}=1$ and $1<p<\infty$.

TheOREM B. A matrix function $M \in G L_{\infty}$ admits a generalized canonical factorization $M=M_{+} M_{-}$in $L_{p}$ if and only if $T_{M}, R_{M}$ are invertible on $L_{p}, L_{q}$ and then equations (3.3) hold. If $M \in G C$ admits a canonical factorization in $L_{p}$ for some $p, 1<p<\infty$, it admits a canonical factorization in $L_{r}$, for all $1<r<\infty$. That is, $\left(M_{ \pm}\right)^{ \pm 1} \in L_{r}$ for all $1<r<\infty$.

In light of Theorems $\mathrm{A}$ and $\mathrm{B}$ we need to examine the continuity of the operators $T_{M}, R_{M}, \hat{T}_{M}, \hat{R}_{M}$.

THEOREM 3.1. Let $M \in G L_{\infty}$ admit a canonical factorization in $L_{p}$ for some $1<p<\infty$. Then for any $\varepsilon>0$ there exist a $\delta>0$ such that $\|M-N\|_{\infty}<\delta$ implies $N$ has a canonical factorization in $L_{p}$ and

$$
\left\|M_{+}-N_{+}\right\|_{q}<\varepsilon, \quad\left\|M_{-}^{-1}-N_{-}^{-1}\right\|_{q}<\varepsilon
$$




$$
\left\|M_{+}^{-1}-N_{+}^{-1}\right\|_{p}<\varepsilon, \quad\left\|M_{-}-N_{-}\right\|_{p}<\varepsilon,
$$

where $p^{-1}+q^{-1}=1$.

This theorem is known [4], but we will give the proof to note some facts about the operators $T, R$ which will be useful later.

LEMMA 3.1. Let $T_{1}$ be an invertible operator and $\left\|T_{1}-T_{2}\right\|<\left\|T_{1}^{-1}\right\|^{-1}$. Then $T_{2}$ is invertible and

$$
\left\|T_{1}^{-1}-T_{2}^{-1}\right\| \leqslant \frac{\left\|T_{1}^{-1}\right\|^{2}\left\|T_{1}-T_{2}\right\|}{1-\left\|T_{1}^{-1}\right\|\left\|T_{1}-T_{2}\right\|} .
$$

In particular, if $\left\|T_{1}-T_{2}\right\| \leqslant\left(2\left\|T_{1}^{-1}\right\|\right)^{-1}$ then

$$
\left\|T_{1}^{-1}-T_{2}^{-1}\right\| \leqslant 2\left\|T_{1}^{-1}\right\|^{2}\left\|T_{1}-T_{2}\right\| .
$$

Proof. The result is standard; see, for example, [8].

LeMma 3.2. Let $M, N \in G L_{\infty}$ and $1<p<\infty$. Then $\left\|T_{M}-T_{N}\right\| \leqslant K_{1} \| M-$ $N\left\|_{\infty},\right\| R_{m}-R_{N}\left\|\leqslant K_{2}\right\| M-N \|_{\infty}$, and provided $\|M-N\|_{\infty}$ is sufficiently small, $\left\|\hat{T}_{M}-\hat{T}_{N}\right\|<K_{3}\|M-N\|_{\infty}, \quad\left\|\hat{R}_{M}-\hat{R}_{N}\right\|<K_{4}\|M-N\|_{\infty}$, where $K_{t}, \quad i=$ $1, \ldots, 4$ are constants, and $\|\cdot\|$ denotes the operator norm induced by $L_{p}$ or $L_{q}$ as appropriate.

Proof. We give the proof for $T, \hat{T}$ as the argument for $R, \hat{R}$ is almost identical.

$$
\begin{aligned}
\left\|T_{M}(X)-T_{N}(X)\right\|_{p} & =\left\|P_{+}(X M-X N)\right\|_{p} \\
& \leqslant\left\|P_{+}\right\|\|X(M-N)\|_{p} \\
& \leqslant\left\|P_{+}\right\|\|X\|_{p}\|M-N\|_{\infty} .
\end{aligned}
$$

Just set $K_{1}=\left\|P_{+}\right\|$and recall $K_{1}<\infty$ as $P_{+}$is bounded on $L_{p}$.

$$
\begin{aligned}
\left\|\hat{T}_{M}(X)-\hat{T}_{N}(X)\right\|_{p} & =\left\|P_{+}\left(M^{-1}-N^{-1}\right) X\right\|_{p} \\
& \leqslant\left\|P_{+}\right\|\|X\|_{p}\left\|M^{-1}-N^{-1}\right\|_{\infty} \\
& \leqslant K_{3}\|X\|_{p}\|M-N\|_{\infty},
\end{aligned}
$$

provided $\|M-N\|_{\infty}$ is sufficiently small.

Proof of Theorem 3.1. We give details only for $\left\|M_{-}^{-1}-N_{-}^{-1}\right\|$, the others following almost identical arguments. Fix $\varepsilon>0$. By Lemma 3.1, $R_{N}$ is invertible 
provided $\left\|R_{M}-R_{N}\right\| \leqslant\left\|R_{M}^{-1}\right\|^{-1}$. Note $R_{M}$ is invertible by Theorem B. By (3.3).

$$
\begin{aligned}
\left\|M_{-}^{-1}-N_{-}^{-1}\right\|_{q} & =\left\|R_{M}^{-1}(I)-R_{N}^{-1}(I)\right\|_{q} \\
& \leqslant\left\|R_{M}^{-1}-R_{N}^{-1}\right\|\|I\|_{q} \\
& \leqslant 2\left\|R_{M}^{-1}\right\|^{2}\left\|R_{M}-R_{N}\right\|\|I\|_{q} \text { for }\left\|R_{M}-R_{N}\right\| \leqslant \frac{1}{2}\left\|R_{M}^{-1}\right\|^{-1} \\
& \leqslant 2\left\|\bar{K}_{M}^{\prime}\right\|^{2}\|\bar{I}\|_{q} \bar{K}_{2}\|\bar{M}-\bar{N}\|_{\infty} .
\end{aligned}
$$

So choose

$$
\delta_{1}=\frac{1}{2 K_{2}\left\|R_{M}^{-1}\right\|\|I\|_{q}} \min \left(1, \frac{\varepsilon}{\left\|R_{M}^{-1}\right\|}\right) .
$$

For $M_{+}, N_{-}, M_{+}^{-1}$ we get similarly $\delta_{2}, \delta_{3}, \delta_{4}$ and set $\delta<\min \left(\delta_{1}, \delta_{2}, \delta_{3}, \delta_{4}\right)$.

From a control systems viewpoint, Theorem 3.1 is of limited value. Consider, for example, the problem of controller design using linear-quadratic-gaussianmethods [3]. There is given the transfer-function matrix of a plant, and from this one calculates a controller transfer-function matrix. The controller is implemented, perhaps on a computer, and connected to the plant. Among the various requirements on the interconnected (closed loop) system is stability (freedom from unbounded signals). The calculation procedure leading to the controller uses two spectral factorizations, the "spectra" being determined by the plant transfer matrix. However, the transfer function is never known exactly, since it is only in some way an approximation to the physical plant. It follows that the controller can be in error.

The stability requirement for the plant-controller interconnection is one which, at least in the scalar case (i.e. $1 \times 1$ case) has a graphical interpretation. One defines from the plant and controller a loop-gain transfer function and studies, for the purpose of assessing stability, the number of encirclements of the point -1 in the complex plane of the graph of this transfer function, viewed as a mapping from the unit circle to the complex plane. If the overall mapping from plant to loop gain transfer function is such that a small $L_{\infty}$ variation in the plant can lead to a small $L_{p}, p \neq \infty$, variation in the loop gain (but sizeable $L_{\infty}$ variation), then a small perturbation in the plant can change a stable situation to an unstable one, through a change in the number of encirclements of the -1 point. Because the loop gain in part depends on two spectral factorizations, Theorem 3.1 leaves open this possibility.

This motivates the study of $L_{\infty}$ continuity, which is the subject of Theorem 3.2. The example of [1] however shows that Theorem 3.1 is tight. That is, one cannot extend to the case $p=\infty$ without assumptions on $M$ and $N$ in addition to those 
imposed in Theorem 3.1. The condition we impose is absolute continuity, or equivalently that $M$ and $N$ are indefinite integrals (see e.g. [11]).

Let $S_{a}$ be the "phase shift by $a$ " operator on the space of matrix functions. That is, if $M$ is a matrix function,

$$
\left(S_{a} M\right)\left(e^{j \omega}\right)=M\left(e^{\jmath(\omega+a)}\right) .
$$

Now let $d M$ be the matrix function defined by the pointwise limit, where it exists;

$$
d M\left(e^{j \omega}\right)=\lim _{\Delta \omega \rightarrow 0} \frac{\left(S_{\Delta \omega} M\right)\left(e^{j \omega}\right)-M\left(e^{j \omega}\right)}{\Delta \omega} .
$$

If $M$ is absolutely continuous, then $d M$ exists everywhere, is in $L_{1}$ and the fundamental theorem of calculus holds [11].

THEOREM 3.2. Let $M \in G C \subset G L_{\infty}$ and suppose $M$ has a canonical factorization in $L_{p}, 1<p<\infty$. Further suppose $M$ is absolutely continuous and $d M \in L_{r}$, for some $r>1$. Then for any $\varepsilon>0$ and any $K<\infty$, there exists $a \delta=\delta(K, \varepsilon, M)>0$ such that $N \in L_{\infty}$ and absolutely continuous, $\|M-N\|_{\infty}<\delta, d N \in L_{r}$ and $\|d M-d N\|_{r}<K$ imply $M_{ \pm}, N_{ \pm}, M_{ \pm}^{-1}, N_{ \pm}^{-1} \in G C$. Furthermore

$$
\left\|M_{ \pm}-N_{ \pm}\right\|_{\infty}<\varepsilon, \quad\left\|M_{ \pm}^{-1}-N_{ \pm}^{-1}\right\|_{\infty}<\varepsilon .
$$

The appendix contains a straightforward proof for the scalar spectral factorization case (i.e. $M$ scalar and positive definite). Before proceeding to the more "modern" and independent proof of the full result, we derive a result about the differentiability of the factors $M_{ \pm}, N_{ \pm}$which we will use in the proof.

Lemma 3.3. Let $A \in G C$ admit a canonical factorization in $L_{p}, 1<p<\infty$ and suppose $d A \in L_{r}, r>1$. Then

$$
d A_{+}, d A_{-}^{-1}, d A_{+}^{-1}, d A_{-} \in L_{r} .
$$

Furthermore

$$
\begin{aligned}
& d A_{+}^{-1}=-T_{A}^{-1} P_{+}\left(A_{+}^{-1} d A\right) \\
& d A_{-}^{-1}=-R_{A}^{-1} P_{-}\left(d A A_{-}^{-1}\right) \\
& d A_{+}=-\hat{T}_{A}^{-1} P_{+}\left(d A^{-1} A_{+}\right) \\
& d A_{-}=-\hat{R}_{A}^{-1} P_{-}\left(A_{-} d A^{-1}\right)
\end{aligned}
$$

Proof. The four cases are almost identical, so we give the argument only for $A_{+}^{-1}$, i.e. for (3.9a). 
Note that (3.8) follows from (3.9), since by minor variation on Hölder's inequality $A_{+}^{-1} d A \in L_{t}, 1<t<r$, provided $d A \in L_{r}$ and $A_{+}^{-1} \in L_{p}$, where $p=$ $r t(r-t)^{-1}$. By Theorem $B, A_{+}^{-1} \in L_{p}$, for all $1<p<\infty, P_{+}$is bounded on $L_{t}$ and $T_{A}$ is invertible on $L_{t}$. Thus, with $d A_{+}^{-1}$ given by (3.9a), $d A_{+}^{-1} \in L_{t}$ for any $1<t<r$. However, this implies $A_{+}^{-1} \in L_{\infty}$. Repeating the above argument allowing $p=\infty$ gives $d A_{+}^{-1} \in L_{r}$. We now prove (3.9a).

By Theorem B, $T_{A}\left(A_{+}^{-1}\right)=I$. Also, since $S_{\Lambda_{\omega}}$ is a rigid rotation of the unit circle,

$$
T_{S_{\Delta \omega} A}\left(S_{\Delta \omega} A_{+}^{-1}\right)=I
$$

Subtracting and using (3.2a) we obtain, after a trivial manipulation

$$
P_{+}\left[\left(S_{\Delta \omega} A_{+}^{-1}-A_{+}^{-1}\right) S_{\Delta \omega} A\right]+P_{-}\left[S_{\Delta \omega} A_{+}^{-1}-A_{+}^{-1}\right]=-P_{+}\left[A_{+}^{-1}\left(S_{\Delta \omega} A-A\right)\right],
$$

or equivalently

$$
\left(T_{S_{\Delta \omega} A}\right)\left[S_{\Delta \omega} A_{+}^{-1}-A_{+}^{-1}\right]=-P_{+}\left[A_{+}^{-1}\left(S_{\Delta \omega} A-A\right)\right] .
$$

Hence

$$
\frac{S_{\Delta \omega} A_{+}^{-1}-A_{+}^{-1}}{\Delta \omega}=-\left(T_{S_{\Delta \omega} A}\right)^{-1} P_{+}\left[A_{+}^{-1}\left(\frac{S_{\Delta \omega} A-A}{\Delta \omega}\right)\right],
$$

provided $T_{S_{\Delta_{\omega} A}}$ is invertible. However, by Lemma 3.1 we know that for $\| T_{S_{\Delta_{\omega} A}}-$ $T_{A} \|$ sufficiently small, $T_{S_{\Delta \omega} A}$ is invertible, and

$$
\left\|\left(T_{S_{\Delta \omega} A}\right)^{-1}-T_{A}^{-1}\right\| \leqslant K\left\|T_{S_{\Delta \omega} A}-T_{A}\right\| .
$$

Now by Lemma $3.2,\left\|T_{S_{\Delta \omega} A}-T_{A}\right\| \leqslant C\left\|S_{\Delta \omega} A-A\right\|_{\infty} \rightarrow 0$ as $\Delta \omega \rightarrow 0$ by the continuity of $A$ (recall $A \in G C$ ). Thus $\left\|\left(T_{S_{\Delta \omega} A}\right)^{-1}-\left(T_{A}\right)^{-1}\right\| \rightarrow 0$ as $\Delta \omega \rightarrow 0$, and letting $\Delta \omega \rightarrow 0$ in (3.10) we obtain (3.9a).

\section{REMARKS.}

1. If $A_{+}^{-1}$ is differentiable, we can obtain (3.9a) by differentiating the equation $T_{A}\left(A_{+}^{-1}\right)=I$. The main task of the proof is to show $A_{+}^{-1}$ is differentiable. This amounts to showing $\left\|\left(T_{S_{\Delta \omega} A}\right)^{-1}-T_{A}^{-1}\right\| \rightarrow 0$ as $\Delta \omega \rightarrow 0$.

2. The restriction $r>1$, rather than $r \geqslant 1$, is because $P_{+}$is not bounded on $L_{1}$, and $T_{A}$ is not invertible on $L_{1}$.

Proof of Theorem 3.2. By Theorem 3.1, we know that for $\delta$ sufficiently small, $N$ has a generalized canonical factorization in $L_{p}$, for all $1<p<\infty$. Applying Lemma 3.3 to $M$ and $N$ we find that their canonical factors are in $G L_{\infty}$ (indeed $G C)$. 
Again, the argument is almost identical for $M_{+}, M_{-}$, etc., so we give the details only for $\left\|M_{+}^{-1}-N_{+}^{-1}\right\|_{\infty}$.

Let $W=M_{+}^{-1}-N_{+}^{-1}$. By the equivalence of norms on $\mathrm{C}^{n \times n}$, there are constants $K_{1}, K_{2}$ such that

$$
\left(1 / K_{2}\right) \operatorname{tr}\left[W\left(e^{j \omega}\right) W\left(e^{j \omega}\right)^{*}\right] \leqslant\left|W\left(e^{j \omega}\right)\right|^{2} \leqslant K_{1} \operatorname{tr}\left[W\left(e^{j \omega}\right) W\left(e^{j \omega}\right)^{*}\right] .
$$

Hence

$$
\begin{aligned}
\left|W\left(e^{j \omega}\right)\right|^{2} & \leqslant K_{1} \operatorname{tr}\left[W\left(e^{j \omega}\right) W\left(e^{j \omega}\right)^{*}\right] \\
& \leqslant K_{1} \operatorname{tr} \int_{\theta}^{\omega} \frac{d}{d \tau}\left[W\left(e^{j \tau}\right) W\left(e^{j \tau}\right)^{*}\right] d \tau+K_{1} \operatorname{tr} W\left(\left(e^{j \theta}\right) W e^{j \theta}\right)^{*}
\end{aligned}
$$

since $W$ is absolutely continuous. Now let $t \in(1, r)$ and $p^{-1}=1-t^{-1}$. Choose $\theta$ such that

$$
\operatorname{tr}\left[W\left(e^{j \theta}\right) W\left(e^{j \theta}\right)^{*}\right] \leqslant K_{2}\left|W\left(e^{j \theta}\right)\right|^{2} \leqslant K_{2}\|W\|_{p}^{2} .
$$

It is trivial to verify by contradiction that such a $\theta$ exists. Hence we obtain

$$
\begin{aligned}
\left|W\left(e^{j \omega}\right)\right|^{2} \leqslant & K_{1} \int_{\theta}^{\omega} \operatorname{tr}\left[\left(d W\left(e^{j \tau}\right) W e^{j \tau}\right)^{*}\right] d \tau \\
& +K_{1} \int_{\theta}^{\omega} \operatorname{tr}\left[W\left(e^{j \tau}\right) d W\left(e^{j \tau}\right)^{*}\right] d \tau+K_{1} K_{2}\|W\|_{p}^{2} .
\end{aligned}
$$

Now $d W, d W^{*} \in L_{r}$, hence $\in L_{t}$ and $W \in L_{p}, \forall p>1$, so by Hölder's inequality applied to (3.12) we obtain, with $p^{-1}=1-t^{-1}$,

$$
\begin{aligned}
\left|W\left(e^{j \omega}\right)\right|^{2} \leqslant & 2 K_{1}\left(\int_{\theta}^{\omega} \operatorname{tr}\left[d W\left(e^{j \tau}\right) d W\left(e^{j \tau}\right)^{*}\right]^{t / 2} d \tau\right)^{1 / t} \\
& \times\left(\int_{\theta}^{\omega} \operatorname{tr}\left[W\left(e^{j \tau}\right) W\left(e^{j \tau}\right)^{*}\right]^{p / 2} d \tau\right)^{1 / p} \\
& +K_{1} K_{2}\|W\|_{p}^{2} \\
\leqslant & 2 K_{1} K_{2}\left(\int_{\theta}^{\omega}\left|d W\left(e^{j \tau}\right)\right|^{t} d \tau\right)^{1 / t}\left(\int_{\theta}^{\omega}\left|W\left(e^{j \tau}\right)\right|^{p} d \tau\right)^{1 / p} \\
& +K_{1} K_{2}\|W\|_{p}^{2} \\
\leqslant & K_{1} K_{2}\left(2\|d W\|_{t}+\|W\|_{p}\right)\|W\|_{p} .
\end{aligned}
$$

Provided $\|d W\|_{t}$ is bounded, the result will follow from Theorem 3.1 by choosing $\delta$ such that $\|W\|_{p}$ is small enough to make the right hand side of (3.13) less than 
$\varepsilon$. The boundedness of $\|d W\|_{t}$ follows from Lemma 3.2 as follows:

$$
\begin{aligned}
\|d W\|_{t} & =\left\|d M_{+}^{-1}-d N_{+}^{-1}\right\|_{t} \leqslant\left\|d M_{+}^{-1}\right\|_{t}+\left\|d N_{+}^{-1}\right\|_{t} \\
& \leqslant\left\|T_{M}^{-1} P_{+}\left(M_{+}^{-1} d M\right)\right\|_{t}+\left\|T_{N}^{-1} P_{+}\left(N_{+}^{-1} d N\right)\right\|_{t} \\
& \leqslant\left\|T_{M}^{-1}\right\|\left\|P_{+}\right\|\left\|M_{+}^{-1} d M\right\|_{t}+\left\|T_{N}^{-1}\right\|\left\|P_{+}\right\|\left\|N_{+}^{-1} d N\right\|_{t} \\
& \leqslant\left\|T_{M}^{-1}\right\|\left\|P_{+}\right\|\left\|M_{+}^{-1}\right\|_{q}\|d M\|_{\tau}+\left\|T_{N}^{-1}\right\|\left\|P_{+}\right\|\left\|_{\|} N_{+}^{-1}\right\|_{q}\|d N\|_{r}, \\
& q=r t(r-t)^{-1}
\end{aligned}
$$

$\leqslant C$,

by Theorem 3.1. Note $C$ depends on $\left\|M_{+}^{-1}\right\|_{q},\left\|M_{+}^{-1}-N_{+}^{-1}\right\|_{q}$ (bounded by Theorem 3.1), $\|d M\|_{r}$ and $\|d M-d N\|_{r}$ (<K by hypothesis).

\section{REMARKS.}

1. The Theorem does not impose a smallness assumption on $\|d M-d N\|_{r}$. That is, $K$ is not dependent on $\varepsilon$. Naturally a smaller $K$ will lead to a more generous $\delta$. In other words, for a given $\varepsilon, \delta(\varepsilon, K)$ is a decreasing function of $K$.

2. If $M, N$ are positive definite Hermitian, it is easy to show [9] that $M_{+}=M_{-}^{*}$, and then Theorems 3.1 and 3.2 specialize to the corresponding spectral factorization results in [1].

3. Any rational matrix function with no poles on the unit circle is absolutely continuous on the unit circle and has $L_{r}$ derivatives for all $1 \leqslant r \leqslant \infty$.

\section{Continuity of Wiener-Hopf factorization}

In this section we generalize, as far as is possible, Theorem 3.2 to Wiener-Hopf (W-H) factorization. We first however, review briefly this factorization and note some properties which will limit the generalization. The review material comes from $[4,9]$.

A matrix function $M \in G W$ is said to admit a (left) Wiener-Hopf (W-H) factorization in case

$$
M=M_{+} D M_{-},
$$

where $M_{ \pm} \in G W^{ \pm}, M_{-}(\infty)=I$ and either

$$
D(z)=\operatorname{diag}\left(\left(\frac{z-z_{+}}{z-z_{-}}\right)^{k_{1}} i=1, \ldots, n\right), \quad k_{i} \geqslant k_{k+1},
$$

with $\left|z_{+}\right|<1,\left|z_{-}\right|>1$ arbitrary and $k_{i}$ integers, called the partial indices of $M$, or

$$
D(z)=\operatorname{diag}\left(z^{k_{x}}, i=1, \ldots, n\right),
$$

in which case the factorization is called standard. 
THEOREM D. Every $M \in G W$ admits a $W-H$ factorization, and the partial indices are unique.

We can also discuss generalized W-H factorization in $L_{p}$ of matrix functions in $G L_{\infty}$ or $G C$, where $D$ is as in (4.2) and $M_{ \pm}$satisfy the same conditions as for canonical factorization in $L_{p}$.

TheOREM E. Every $M \in G C$ admits a generalized $W$ - $H$ factorization in $L_{p}$ for every $1<p<\infty$.

A matrix function $M \in G C$ will be said to have stable indices if there is a $\delta>0$ such that for any $N \in G C$ satisfying $\|M-N\|_{\infty}<\delta, M$ and $N$ have the same partial indices.

The major difficulty in extending the theorems of Section 3 to $\mathrm{W}-\mathrm{H}$ factorization in $L_{p}$ is the following result $[4,9]$.

Let $S_{n}$ denote the set of ordered sequences $\left\{k_{i}\right\}$ of $n$ integers with $k_{i} \geqslant k_{i+1}$, $i=1, \ldots, n$. Suppose $\left\{k_{i}\right\}$ and $\left\{k_{i}^{\prime}\right\} \in S_{n}$. Define a partial order $>$ by

$$
\left\{k_{i}\right\} \succ\left\{k_{i}^{\prime}\right\} \text { if } \sum_{j=1}^{i} k_{j} \geqslant \sum_{j=1}^{i} k_{J}^{\prime}, i=1, \ldots, n-1
$$

and

$$
\sum_{j=1}^{n} k_{j}=\sum_{j=1}^{n} k_{j}^{\prime}
$$

Further, define the "average" of an element $\left\{k_{i}\right\}$ of $S_{n}$, denoted $\left\{\hat{k}_{i}\right\}$

$$
\begin{aligned}
\hat{k}_{i} & =q+1, & & i=1, \ldots, r, \\
\hat{k}_{i} & =q, & i & =r+1, \ldots, n,
\end{aligned}
$$

where

$$
\sum_{j=1}^{n} k_{j}=n q+r, \quad 0 \leqslant r<n .
$$

The average has the "minimality" property that if $\left\{k_{i}^{\prime}\right\} \in S_{n}$ and $\left\{k_{i}\right\} \succ\left\{k_{i}^{\prime}\right\}$, then $\left\{k_{i}^{\prime}\right\}>\left\{\hat{k}_{i}\right\}$.

THEOREM F. Let $M \in G L_{\infty}$ (or $G C$ ) admit a generalized $W$-H factorization in $L_{p}$, some $1<p<\infty$. Then there exists a $\delta>0$ such that for any $N \in G L_{\infty}$, $\|M-N\|_{\infty}<\delta$ the following "inequality" holds:

$$
\left\{k_{i}(M)\right\}>\left\{k_{i}(N)\right\}>\left\{\hat{k}_{i}(M)\right\}
$$


where $\left\{k_{i}(A)\right\}$ denotes the partial indices of a matrix function $A$. Conversely, let $\left\{\tilde{k}_{i}\right\}$ be arbitrary in $S_{n}$ with $\left\{k_{i}(M)\right\} \succ\left\{\tilde{k}_{i}\right\}$. Then, for any $\delta>0$, there exists an $N \in G L_{\infty}(G C)$, such that $\|M-N\|<\delta$ and $k_{i}(N)=\tilde{k}_{i}, i=1, \ldots, n$.

The difficulty in extending the theorems of Section 3 to the general W-H factorization is because of the extremely undesirable properties of partial indices -c.f. the converse statement in Theorem F.

It is very easy to deduce the following corollary.

Corollary G. $M \in G L_{\infty}$ has stable indices if and only if $k_{i}(M)=\hat{k}_{i}(M)$, $i=1, \ldots, n$. Equivalently, $k_{1} \leqslant k_{n}+1$, i.e. there is a difference of 1 or 0 between the largest and smallest indices.

REMARKS. If all the indices of $M$ are zero (i.e. $M$ has a canonical factorization) then obviously $M$ has stable indices.

In the light of Theorem $F$, it is unreasonable to expect the factorization operation to be continuous (in any norm) unless one has a matrix with stable indices. Note that by Theorem F, there is a matrix function with stable indices arbitrarily close (in $L_{\infty}$ norm) to any factorizable matrix function. Stable indices are the generic case, indeed matrix functions with stable indices are dense in $G L_{\infty}$, with $L_{\infty}$ norm. Thus we generalize below Theorem 3 to matrix function $M \in G C$ with stable indices.

By way of motivation, let us note an application in system theory. Errors-invariables identification [2] is the name given to a class of procedures for passing from noisy measurements of the input and output signals of a linear system characterized by an unknown convolution operator, to a description of that system (convolution operator or transfer function). The procedure of [10] uses W-H factorization of a matrix function, which is a sub-matrix of the power spectrum matrix associated with the measured signals, to calculate the convolution operator. The determination of the power spectrum matrix can never be totally error free, and so the factors resulting from the W-H factorization will be in error. Since these factors are used to determine the unknown convolution operator the calculated convolution operator will inherit errors. It is important that these errors be controlled in an $L_{\infty}$ sense, particularly if a controller is subsequently to be connected to the identified system, as noted previously.

The next theorem provides conditions for the $L_{\infty}$ continuity.

TheOREM 4.1. Let $M \in G C$ be absolutely continuous and suppose $d M \in L_{r}$ for some $r>1$. Further suppose $M$ has stable partial indices. Then for any $\varepsilon>0$ and any $K<\infty$, there exists a $\delta=\delta(K, \varepsilon, M)$ such that if $N$ is absolutely continuous, $d N \in L_{r},\|M-N\|_{\infty}<\delta$, and $\|d M-d N\|_{r}<K$, then $M_{ \pm}, N_{ \pm}, M_{ \pm}^{-1} \in G C$. 
Furthermore

$$
\left\|M_{ \pm}-N_{ \pm}\right\|_{\infty}<\varepsilon, \quad\left\|M_{ \pm}^{-1}-N_{ \pm}^{-1}\right\|_{\infty}<\varepsilon
$$

To prove Theorem 4.1, we shall make use of the following result of [4].

THEOREM H. Every element $A \in G C$ can be factored in the form $A=D_{0} A_{0}$, where $A_{0} \in G C$ admits a left canonical factorization in $L_{p}, 1<p<\infty$, and $D_{0}(t)=\operatorname{diag}\left(z^{\tau} i, i=1, \ldots, n\right)$ with $\tau_{i}$ a permutation of the set $\left\{k_{i}(A)\right\}$ of partial indices of $A$. Moreover if $A=A_{+} D A_{-}, A_{0}$ may be factored as $A_{0}=\tilde{A}_{+} A_{-}$.

Proof of Theorem 4.1. That $N$ has a factorization for $\delta$ sufficiently small follows from Theorem E, since $N \in C$ and $\|M-N\|_{\infty}$ sufficiently small imply $N \in G C$. Also, since $M$ has stable partial indices, we can choose $\delta$ small enough for $N$ to have the same indices as $M$, by Theorem $\mathrm{F}$. Then

$$
\begin{aligned}
M & =M_{+} D M_{-}=D_{0} \tilde{M}_{+} M_{-}=D_{0} M_{0}, \\
N & =N_{+} D N_{-}=D_{1} \tilde{N}_{+} N_{-}=D_{1} N_{0} .
\end{aligned}
$$

We must now show that for $\delta$ sufficiently small, $D_{1}=D_{0}$. This depends on an analysis of the proof of Theorem $\mathrm{H}$ (see (see [4], pp. 128-131). Briefly, the indices are reordered in going from $D$ to $D_{1}$ (or $D_{0}$ ) on the basis of certain minors of $N_{+}(\infty)$ (resp. $M_{+}(\infty)$ ) being non-zero, and on the solution of linear equations derived from $M_{+}(\infty)$ (resp. $N_{+}$)). Thus if $\delta$ is sufficiently small, the reordering can be chosen to be the same for $N$ and $M$, i.e. we may choose $D_{0}=D_{1}$ provided $\delta$ is sufficiently small. Then

$$
\begin{aligned}
\left\|M_{0}-N_{0}\right\|_{\infty} & =\left\|D_{0}^{-1}(M-N)\right\|_{\infty} \\
& \leqslant\left\|D_{0}^{-1}\right\|_{\infty}\|M-N\|_{\infty} \\
& \leqslant K_{1}\|M-N\|_{\infty}, \text { since }\left\|D^{-1}\right\|_{\infty}=K_{1}<\infty .
\end{aligned}
$$

Thus we can make $\left\|M_{0}-N_{0}\right\|$ as small as we like. Moreover, $M_{0}$ and $N_{0}$ are clearly absolutely continuous, since $M, N$ and $D_{0}$ are, and their derivatives are in $L_{r}$. Thus by Theorem 3.2 applied to $M_{0}, N_{0}$, for $\delta$ sufficiently small,

$$
\left\|M_{-}-N_{-}\right\|_{\infty}<\varepsilon, \quad\left\|M_{-}^{-1}-N_{-}^{-1}\right\|_{\infty}<\varepsilon .
$$

Furthermore,

$$
\begin{aligned}
\left\|M_{+}-N_{+}\right\|_{\infty} & =\left\|\left(M M_{-}^{-1}-N N_{-}^{-1}\right) D^{-1}\right\|_{\infty} \\
& \leqslant\left\|\left(M M_{-}^{-1}-N N_{-}^{-1}\right)\right\|_{\infty}\left\|D^{-1}\right\|_{\infty} \\
& \leqslant\left[\left\|M\left(M_{-}^{-1}-N_{-}^{-1}\right)\right\|_{\infty}+\left\|(M-N) N_{-}^{-1}\right\|_{\infty}\right]\left\|D^{-1}\right\|_{\infty} \\
& \leqslant\left[\|M\|_{\infty}\left\|M_{-}^{-1}-N_{-}^{-1}\right\|_{\infty}+\|M-N\|_{\infty}\left\|N_{-}^{-1}\right\|_{\infty}\right]\left\|D^{-1}\right\|_{\infty}
\end{aligned}
$$


and it follows that $\left\|M_{+}-N_{+}\right\|_{\infty}<\varepsilon$ for $\delta$ sufficiently small. Also

$$
\begin{aligned}
\left\|M_{+}^{-1}-N_{+}^{-1}\right\| & =\left\|D M_{-} M^{-1}-D N_{-} M^{-1}\right\|_{\infty} \\
& =\left\|D\left[\left(M_{-}-N_{-}\right) M^{-1}+N_{-}\left(M^{-1}-N^{-1}\right)\right]\right\|_{\infty} \\
& \leqslant\|D\|_{\infty}\left[\left\|M_{-}-N_{-}\right\|_{\infty}\left\|M^{-1}\right\|_{\infty}+\left\|N_{-}\right\|_{\infty}\left\|M^{-1}-N^{-1}\right\|_{0}\right],
\end{aligned}
$$

so $\|_{i} M_{+}^{-1}-N_{+}^{-1} i_{\infty}<\varepsilon$ for ô sufficientily small. The theorem is proved.

REMARK. Let $S$ be an algebra of functions. If every $F \in G S$ has a W $-\mathrm{H}$ factorization, with the spaces containing the factors $F_{ \pm}$subsets of $S$, the factorization is called proper (this is called canonical factorization in [7]). Factorization in $W$ is proper, since $W^{ \pm} \subset W$. Generalized factorization of $G L_{\infty}$ or $G C$ functions in $L_{p}$ is not proper $\left(L_{p, q} \not \subset L_{\infty}, q^{-1}+p^{-1}=1,1<p<\infty\right)$. If we define $C^{r}=\left\{A \in C\right.$ such that $A$ is absolutely continuous and $d A \in L_{r}$, $r>1\}$, then Lemma 3.3 and Theorem $\mathrm{H}$ give the following:

\section{COROLlaRY 4.2. Factorization of matrix functions in $G C^{r}$ is proper.}

Proof. Reduce to the canonical case using Theorem $\mathrm{H}$ and the result follows from Lemma 3.3 and elementary manipulations. Remember $D^{ \pm 1} \in C^{r}$.

It is known that if $A \subset C$ is a Banach algebra, then factorization in $G A$ is proper if and only if $A$ is decomposing, or equivalently if $P_{+}$is bounded on $A$ [4, 7]. $W$ is decomposing, since $W=W^{+} \oplus W^{-}$, but $C$ and $L_{\infty}$ are not $\left(P_{+}\right.$is not bounded in $L_{\infty}$ ). In a decomposing algebra, the continuity of the factorization follows directly from the boundedness (hence continuity) of $P_{+}$. However $C^{r}$ is not decomposing and $P_{+}$is not bounded on $C^{r}$ (in $L_{\infty}$ norm), even though factorization is proper in $C^{r}$. The problem is that $C^{r}$, with $L_{\infty}$ norm, is not a Banach algebra. In fact it is a non-closed sub-algebra of the Banach algebra $C$. The bound $\|d M-d N\|_{r}<K$ in Theorems 3.2, and 4.1 is to counter the non-closed nature of $C^{r}$. It prevents the situation of a sequence $\left\{N_{n}\right\}$ in $C^{r}$ such that $\left\|M-N_{n}\right\|_{\infty}<\delta$ for all $n$, but $\left\|d M-d N_{n}\right\|_{r} \rightarrow \infty$ as $n \rightarrow \infty$ from being considered by Theorems 3.2 and 4.1. In this case $\left\{N_{n}\right\}$ is escaping from $C^{r}$ : it has a limit point in $L_{\infty}$ but not in $C^{r}$. This is the situation in the example in [1]. If $C^{r}$ is equipped with the norm $\|M\|=\|M\|_{\infty}+\|d M\|_{r}$, it becomes a Banach algebra. Hence it is decomposing and $P_{+}$is bounded, since factorization is proper in $C_{r}$. This leads to a different continuity result, namely:

Corollary 4.3. Let $M \in G C^{r}$, some $r>1$, have a $W$-H factorization with stable partial indices. Then for any $\varepsilon>0$ there exist a $\delta>0$ such that if $N \in C^{r}$ 
and $\|M-N\|<\delta$ then

$$
\left\|M_{ \pm}-N_{ \pm}\right\|<\varepsilon \text { and }\left\|M_{ \pm}^{-1}-N_{ \pm}^{-1}\right\|<\varepsilon,
$$

where for $A \in C^{r},\|A\|=\|A\|_{\infty}+\|d A\|_{r}$.

Proof. $\left(C^{r},\|\cdot\|\right)$ is a Banach sub-algebra of $C$. Factorization is proper in $C^{r}$. Thus $P_{+}$is continuous on $\left(C^{r},\|\cdot\|\right)$ and consequently so are the operators $T, R$, $\hat{T}, \hat{R}$ defined in Section 3 . The result follows.

\section{REMARKS.}

1. This result requires the derivatives $d M$ and $d N$ to be close, and implies that $d M_{+}$and $d N_{+}$are close. It thus has more restrictive assumptions and a stronger conclusion than Theorems 3.2 and 4.1 .

2. The continuity of $P_{+}$on $C^{r}$ can be proved without reference to factorization ideas. However, the essential ideas for an independent proof are contained in the proofs of Lemma 3.3 and Theorem 3.2.

\section{Conclusion}

The key conclusion of this paper is that small $L_{\infty}$ perturbations in a matrix function will lead to small $L_{\infty}$ perturbations in its Wiener-Hopf factors provided the factorization indices are stable and the matrix functions (i.e. the original and perturbed) have derivatives in $L_{r}$, for some $r>1$. This condition is seen to be tight by consideration of the example in [1].

The result is seen to be important in assessing the continuity (error containment) properties of the many procedures in system and control theory which use Wiener-Hopf factorization. These include spectral factorization, LQG controller design [3], multiplier theory [5], and errors-in-variables identification [10].

A more desirable property for the above applications would be a "local $L_{\infty}$ continuity property", or "localization principle" for Wiener-Hopf factorization. That is, that small perturbations of a matrix function "mostly" on a subinterval of $[0,2 \pi]$ will produce perturbations of the Wiener-Hopf factors "mostly" in that subinterval. This property is conjectured on the basis of scalar spectral factorization evidence (see appendix). Such a result would be extremely desirable for system theoretic applications since it would mean, for example, that the often significant high frequency errors of a measured power spectrum matrix, arising because of sensor limitations, would not produce large low frequency errors in the calculated Wiener-Hopf (or spectral) factors. 


\section{Appendix}

The purpose of this appendix is to provide what may be a more intuitive proof of Theorem 3.2 for an important special case. The special case is scalar spectral factorization, where one can use the Bode gain/phase relationships to construct the spectral factors. The conditions imposed in Theorem 3.2 are related to properties of these gain/phase relationships which we will derive. This method of proof also leads to the conjecture of a localization principle for scalar spectral factorization, as was mentioned in the conclusion.

ThEOREM A1. Let $\phi_{1}\left(e^{j \omega}\right)=\sum_{-\infty}^{\infty} a_{k} e^{j k \omega}, a_{k}=a_{-k}$, real, $k=0,1, \ldots$ be $a$ positive definite scalar spectrum which is absolutely continuous with derivative $d \phi_{1}$ in $L_{r}[-\pi, \pi]$, some $1<r \leqslant \infty$. Now let $V_{1}\left(e^{j \omega}\right)$ be the stable minimum phase spectral factor of $\phi_{1}$, that is $V_{1}\left(e^{j \omega}\right)$ is the evaluation on $|z|=1$ of a function $V_{1}(z)$ which is analytic and non-zero in $|z| \leqslant 1$ and satisfies

$$
V_{1}\left(e^{j \omega}\right) V_{1}\left(e^{j \omega}\right)^{*}=\phi_{1}\left(e^{j \omega}\right) .
$$

For any $\varepsilon>0$ and any $K<\infty$, there exists $a \delta=\delta\left(\phi_{1}, K, \varepsilon\right)>0$ such that for any real spectrum $\phi_{2}$ such that

$$
\left\|\phi_{1}-\phi_{2}\right\|_{\infty}<\delta \text { and }\left\|d \phi_{1}-d \phi_{2}\right\|_{r}<K \text {, }
$$

then

$$
\left\|V_{1}-V_{2}\right\|_{\infty}<\varepsilon
$$

where $V_{2}$ is the spectral factor of $\phi_{2}$.

Note that this is a very special case of Theorem 3.2. Since the proof by "classical" methods is lengthy in detail, but straightforward, we will give an outline of the main ideas.

OUtLine of Proof. Let $V_{i}\left(e^{j \omega}\right)=R_{i}\left(e^{j \omega}\right) \exp \left\{j I_{i}\left(e^{j \omega}\right)\right\}$ where $R_{i}, I_{i}$ are real valued and

$$
0<R_{i}\left(e^{j \omega}\right)=R_{i}\left(e^{-j \omega}\right), \quad I_{i}\left(e^{j \omega}\right)=-I_{i}\left(e^{-j \omega}\right), \quad i=1,2
$$

It is easy to verify that

$$
\left\|V_{1}-V_{2}\right\|_{\infty} \leqslant\left\|R_{1}-R_{2}\right\|_{\infty}+\left\|R_{1}\right\|_{\infty}\left\|I_{1}-I_{2}\right\|_{\infty} .
$$

So we want to choose $\delta$ such that $\left\|R_{1}-R_{2}\right\|_{\infty}<\varepsilon / 2$ and $\left\|R_{1}\right\|\left\|I_{1}-I_{2}\right\|_{\infty}<\varepsilon / 2$.

Observe the formula $V_{i} V_{t}^{*}=\phi_{i}$ implies $\phi_{t}=R_{t}^{2}$. Hence

$$
\left\|\phi_{1}-\phi_{2}\right\|_{\infty}<\delta \Rightarrow\left\|R_{1}-R_{2}\right\|_{\infty}<\delta^{1 / 2},
$$


since

$$
\begin{aligned}
\left\|R_{1}-R_{2}\right\|_{\infty}^{2} & =\left\|\left(R_{1}-R_{2}\right)^{2}\right\|_{\infty} \\
& \leqslant\left\|\left(R_{1}-R_{2}\right)\left(R_{1}+R_{2}\right)\right\|_{\infty} \text { as } R_{1}, R_{2}>0 \\
& =\left\|\phi_{1}-\phi_{2}\right\|_{\infty} .
\end{aligned}
$$

The problem is thus the $\left\|I_{1}-I_{2}\right\|_{\infty}$ term. Observe $\ln V_{1}=\ln R_{i}+j I_{i}$. Now we use the Bode gain/phase relation to construct $I_{i}$ from $\ln R_{t}=1 / 2 \ln \phi_{i}$.

$$
I_{i}\left(e^{j \omega}\right)^{‘} \frac{1}{2} \int_{-\pi}^{\pi} \frac{\ln \phi_{i}\left(e^{j \omega}\right)-\ln \phi_{i}\left(e^{j \theta}\right)}{\cos \omega-\cos \theta} d \omega,
$$

or, since $\phi_{l}$ is differentiable with integrable derivative, we may integrate by parts to obtain

$$
I_{i}\left(e^{j \theta}\right)=\frac{1}{2} \int_{-\pi}^{\pi} \frac{d \phi_{i}\left(e^{j \omega}\right)}{\phi_{i}\left(e^{j \omega}\right)} \ln \left(\left|\frac{\sin (\theta+\omega) / 2}{\sin (\theta-\omega) / 2}\right|\right) d \omega .
$$

Formula (A.1) has the advantage of not requiring derivatives, but the serious disadvantage that it is not a bounded operator from $L_{\infty}$ to $L_{\infty}$, i.e. small $\phi_{i}$ will not necessarily give small $I_{i}$. An example is given in [1]. Fortunately, (A.2) is a bounded operator from $L_{r}$ to $L_{\infty}$ (for any $r>1$ ). To prove this, one must show that

$$
\sup _{\theta \in[-\pi, \pi)} \int_{-\pi}^{\pi}|\ln | \frac{\sin (\theta+\omega) / 2}{\sin (\theta-\omega) / 2}||^{r} d \omega \leqslant M_{r}<\infty .
$$

This is not completely trivial, but is straightforward using the fact that

$$
|\ln | \frac{\sin (\theta+\omega) / 2}{\sin (\theta-\omega) / 2}||^{r}|\theta-\omega|^{q}|\theta+\omega|^{q} \rightarrow 0 \text { as } \omega \rightarrow \pm \theta \text { for any } r>1, q>0 \text {. }
$$

Then use Hölder's inequality to show $\int_{-\pi}^{\pi} d \omega /\left[\left.|\theta-\omega|\right|^{q}|\theta+\omega|^{q}\right]$ is bounded for some $0<q<1$ (take $q=\frac{1}{4}$ ). This proves (A.3).

We now break the integral in (A.1) up into three parts: one integral over $\left[\theta-\delta_{1}, \theta+\delta_{1}\right]$, one over $\left[-\theta-\delta_{1},-\theta+\delta_{1}\right]$, and the rest. The rest is easy, since so long as $\omega$ is bounded away from $\theta$ (as we have just made sure) the integrand in (A.1) is bounded, so we can choose $\delta$ small enough so that this term is small (say less than $\left.\varepsilon\left(6\left\|R_{1}\right\|_{\infty}\right)^{-1}\right)$.

For the small intervals near $\pm \theta$, we integrate by parts to get the integral in (A.2), but over the small intervals $\left[ \pm \theta-\delta_{1}, \pm \theta+\delta_{1}\right]$, plus the end point evaluation term. The bound (A.3) means that we can choose $\delta_{1}$ small enough for the integral term to be small (say less than $\left.\varepsilon\left(6\left\|R_{1}\right\|\right)^{-1}\right)$, since by hypothesis $\left\|d \phi_{1}-d \phi_{2}\right\|_{r}<K$. Finally, we need to consider the integrated term (from the integration by parts). This causes no problem except when $\theta$ is near zero (in fact, 
when $\left.\theta \in\left[-\delta_{1}, \delta_{1}\right]\right)$, or $\pi$ or $-\pi$. This is because the singularity $\pm \theta$ can coincide with an end point. However, we now simply increase the interval of integration to $\left[-3 \delta_{1}, 3 \delta_{1}\right]$ to avoid the problem.

Notice that because we can use formula (A.1) except over the small intervals, we do not need the derivative bound $K$ to be small. It can be any finite number, and need not tend to zero as $\varepsilon$ tends to zero.

The conjecture of a "local $L_{\infty}$ continuity" result, or "localization principle" derives from the observation that since the integrands of (A.1), (A.2) have singularities at $\theta$, the value of the spectrum $\phi$ at $\theta$ will have a much greater effect on the value of $V$ at $\theta$ than will values of $\phi$ away from $\theta$. That is, the value of $\phi$ at $\theta$ is "overweighted" in (A.1), (A.2), and consequently in $V(\theta)$.

Consequently "localized errors" in $\phi$ should lead to "localized errors" in $V$. This is what we mean by "local continuity".

From an applications viewpoint the desirability of such a result is obvious. In fact it is required if many practical applications of spectral or other Wiener-Hopf factorizations are to be justified-otherwise (for example) high frequency model errors could lead to completely erroneous low frequency LQG controller behaviour. The fact that this does not appear to happen provides circumstantial evidence for the localization principle.

\section{References}

[1] B. D. O. Anderson, "Continuity of the matrix spectral factorization operation", Mat. Appl. Comput. (to appear).

[2] B. D. O. Anderson and M. Gevers, "Identifiability of linear stochastic systems operating under linear feedback", A utomatica 18 (1982), 195-213.

[3] B. D. O. Anderson and J. B. Moore, Linear Optimal Control (Prentice Hall, Englewood Cliffs, N. J., 1971).

[4] K. Clancey and I. Gohberg, Factorization of Matrix Functions and Singular Integral Operators (Birkhauser, Verlag, Basel, 1981).

[5] C. Desoer and M. Vidyasagar, Feedback Systems: Input-Output Properties (Academic Press, New York, 1975).

[6] B. A. Francis, "On robustness of the stability of feedback systems", IEEE Trans. Automat. Control, 25 (1980), 817-818.

[7] I. Gohberg and I. Fel'dman, "Convolution Equations and Projection Methods for their Solution", Amer. Math. Soc. Transl. 1974.

[8] I. Gohberg and S. Goldberg, Basic Operator Theory (Birkhauser, Verlag, Basel, 1981).

[9] I. Gohberg and M. Krein, "Systems of integral equations on a half line with kernels depending on the difference of arguments", Uspekhi Mat. Nauk, 13, No. 2 (80) (1958), 3-72; Amer. Math. Soc. Transl. 2 (1960), 217-287.

[10] M. Green and B. D. O. Anderson, "Identification of multivariable errors-in-variables models with dynamics", IEEE Trans. Automat. Control. 31 (1986), 467-471. 
[11] H. Royden, Real Analysis (Macmillan, New York, 1963).

[12] Y. A. Rozanov, Stationary Random Processes (Holden-Day, San Francisco, 1963).

[13] N. Wiener, The Fourier Integral and Certain of its Applications (Cambridge University Press, London, 1933).

[14] N. Wiener and P. Masani, "The prediction theory of multivariate stochastic processes, parts 1 and 2", Acta Math. 98 (1957), 111-150; 99 (1958), 93-137.

[15] D. C. Youla, "On the factorization of rational matrices", IEEE Trans. Inform. Theory, 17 (1961), 172-189. 